\title{
VALORACIÓN DE PYMES POR DESCUENTO DE FLUJOS DE FONDOS: BASES FILOSÓFICAS Y EPISTEMOLÓGICAS
}

\author{
SMALL AND MEDIUM COMPANY VALUATION BY DISCOUNTED CASH FLOW: \\ PHILOSOPHICAL AND EPISTEMOLOGICAL BASIS
}

German Alfredo Boza Pró Universidad del Pacífico Lima, Perú ORCID: https://orcid.org/0000-0002-5146-4978 Correo electrónico: gboza@valuepartners.pe

Jeri Gloria Ramón Ruffner de Vega Universidad Nacional Mayor de San Marcos Lima, Perú

ORCID: https://orcid.org/0000-0002-5951-6197 Correo electrónico: jramonr@unmsm.edu.pe

\section{RESUMEN}

La mayoría de expertos reconoce al descuento de flujo de fondos como la mejor técnica para valorar las pequeñas y medianas empresas (Pymes). Sin embargo, existe divergencia respecto de los conceptos y criterios que debe incorporar un modelo de valoración. Las finanzas neoclásicas, herederas del positivismo lógico como base epistemológica, mantienen la Hipótesis del Mercado Eficiente (HME) y el capital asset pricing model (CAPM) para calcular la tasa de descuento. Por el contrario, las finanzas conductuales, herederas de la fenomenología de Husserl, sugieren incorporar la conducta o comportamiento humano en sus modelos de valoración. Para tomar postura de uno u otro lado, desde un punto de vista filosófico, un modelo de valoración debe conducirnos al encuentro de la verdad, la justicia y la equidad en la toma de decisiones. Ambos aspectos de la valoración de empresas, el filosófico y el epistemológico, se abordan en este artículo, sobre la base de las diferentes teorías y posiciones vigentes, para culminar con una posición final respecto de los 2 modelos de valoración de empresas actualmente existentes dentro de las finanzas neoclásicas: el Damodaran y el de Fernández.

Palabras clave: Adquisiciones; costo de capital; finanzas; flujos de caja; fusiones; Pymes; valoración.

\begin{abstract}
The majority of experts recognizes the cash flow discount as the best technique to value SMEs. However, there is a divergence in the concepts and criteria that a valuation model must incorporate. neoclassical finance, heiress of logical positivism as an epistemological basis, maintains the efficient market hypothesis (EMH) y the capital asset pricing model (CAPM), to calculate the discount rate. On the contrary, behavioral finance, heiress of Husserl's phenomenology, suggests incorporating human behavior or behavior into its valuation models. On taking position, on one way or another, from a philosophical point of view, a valuation model, should lead us to the encounter of truth, justice and fairness in decision making. Both aspects of company valuation, philosophical and epistemological, are addressed in the present research, analyzing the different theories and positions in force, ending with a final position regarding the 2 valuation models of companies currently existing within Neoclassical Finance: the Damodaran Model and the Fernández Model.
\end{abstract}

Keywords: Acquisitions; cost of capital; finance; cash flow; Mergers; valuation. 


\section{INTRODUCCIÓN}

Al plantear un modelo de valoración de Pymes por descuento de flujo de fondos surgen algunas preguntas fundamentales: ¿Cuál es la razón teleológica (causa final), desde el punto de vista de las ciencias contables y administrativas, de plantear un modelo de valoración de Pymes? ¿Qué consideraciones se deberían tener en cuenta en ese proceso? ¿El modelo de valoración propuesto produce algún tipo de conocimiento nuevo? De ser el caso, ¿cómo se genera este conocimiento y cómo se asegura el camino correcto en la búsqueda de la verdad? ¿Se puede o debe tomar una posición o partido respecto de algún modelo propuesto por los expertos, o más bien se debe tomar una posición neutral? ¿Qué es lo que se está midiendo al proponer una tasa de descuento relevante (coste de capital, $K_{e}$ ) para un modelo de valoración y cómo es que esta tasa se calcula? Finalmente, ¿existe relación entre los conceptos y criterios utilizados por los expertos a la hora de medir el valor de empresa de una Pyme y la búsqueda de la verdad, la justicia y la equidad?

Por un lado, desde un punto de vista filosófico, modelo y valor son palabras claves por analizar y profundizar una propuesta de modelo de valoración. Ellas permiten remitirse a la causa última o finalidad de dicha propuesta. Por otro lado, desde el punto de vista de las ciencias contables y administrativas como disciplina, ¿cuál es la utilidad o fin último de un modelo de valoración de Pymes? Pues es la pertinencia y precisión que debe tener el mismo modelo para ayudar a tomar decisiones, no solo a los directivos de la empresa, sino también a todos sus stakeholders. Los diferentes "grupos de interés" comparten y toman decisiones en función de la información de la empresa y de los modelos o herramientas que tienen a su alcance.

El hecho de que las herramientas de gestión para la toma de decisiones deban ser útiles (es decir, pertinentes y precisas) para más de un interesado en la empresa, remite a una pregunta anterior: ¿cómo logramos acercarnos a la perfección y ser equitativos o justos para todas las partes con la propuesta de un modelo de valoración? Al respecto, Aristóteles propone el uso de la razón práctica o juicio moral en la toma de decisiones.

Por otro lado, desde el punto de vista epistemológico, un modelo de valoración de Pymes debe conducir al encuentro de la verdad y eventualmente a la generación de nuevo conocimiento.

Como puede observarse, diferentes posturas abordan la teoría de conocimiento y cómo se obtiene este. Las finanzas neoclásicas, producto de los paradigmas del positivismo lógico y el racionalismo crítico, se oponen a las finanzas conductuales al desestimar la integración de la subjetividad, la intuición y el comportamiento humano en los modelos de valoración de empresas como pretenden estas últimas. Dentro de un racionalismo más amplio que el positivista, Aristóteles (2014a) propone el uso de la razón práctica para normar nuestras acciones en la construcción de la verdad (un modelo de valoración, en el caso de estudio), reconociendo incluso, que su utilización en la creación de herramientas genera algún tipo de conocimiento.

El presente artículo pretende analizar las diferentes inquietudes filosóficas y epistemológicas que se presentan al proponer un modelo de valoración de empresas con la descripción de las diferentes posiciones doctrinarias existentes sobre el tema y el desarrollo de algunas posibles respuestas. Finalmente, se toma una posición respecto de cada una de ellas y se elige un modelo de valoración de empresas.

\section{El modelo de valoración de empresa como técnica}

Aristóteles (2014a) concibe tres tipos distintitos de actividad humana: la contemplación, regida por la razón teórica; la acción, regida por la razón práctica; y la producción, regida por la razón técnica. Para él la actividad no se opone al pensamiento y, más bien, propone una relación biunívoca de unicidad entre la interacción de ambas actividades.

Según él, de allí se desprenden los diferentes tipos de conocimiento de acuerdo con el objeto de análisis o estudio que se aborde. La razón teórica lleva a las causas y esencias de las cosas. La razón práctica permite discernir entre qué acción tomar (toma de decisiones) ante una disyuntiva, lo que permite que cada uno se ordene y se norme. La primera se aproxima a los medios y los fines; la segunda, al terreno de los medios y se dirige a los resultados (Aristóteles, 2014b, pp. II 2, 994b y VII 1,1028b).

Ahora bien, cuando el Estagirita se refiere al término técni$c a$, alude indistintamente al arte o habilidad que tiene el ser humano para lograr sus metas, por lo que la considera, a su vez, tanto un estado como una facultad. Para Aristóteles (2014a, p. 1140a), la técnica no tiene necesariamente que llevar a lo correcto o moralmente bueno. La técnica está hecha para alcanzar sus metas y objetivos, independientemente de que estas sean mejores o peores (malas o buenas). De allí que la razón técnica necesite de la razón moral para poder discernir entre lo bueno o malo en el uso de una técnica determinada para un fin específico.

De acuerdo con este filósofo, se concluye que las técnicas son moralmente ambiguas, pues es difícil que sean neutrales y, eventualmente, objetivas. Si bien estas proceden de la propia habilidad humana, también son forjadas por los intereses de esa misma naturaleza humana. En este 
sentido, los modelos de valoración de empresas en finanzas, en tanto técnica, no son una excepción.

La tasa de descuento $\left(K_{e}\right)$ en un modelo de valoración de empresas

La fórmula general, en el CAPM, define a la tasa de descuento relevante $\left(K_{e}\right)$ como sigue:

$$
K_{e}=R_{f}+\left(R_{m-} R_{f}\right) \beta
$$

Donde:

$K_{e}$ : rentabilidad exigida a la acción

$R_{f}$ tasa libre de riesgo

$\beta$ : coeficiente beta, riesgo sistémico del sector

$R_{m}-R_{f}$ prima de riesgo del mercado

¿Qué mide la tasa de descuento relevante $\left(K_{e}\right)$ en un modelo de valoración? ¿Qué concepto implícito subyace al calcular esta tasa? ¿Cómo debe abordarse este concepto en un eventual modelo propuesto?

Por simple inspección, se determina que la tasa de descuento $\left(K_{e}\right)$ mide el riesgo de las acciones de una empresa. Todos sus componentes $\left(R_{\rho} \beta\right.$, y $\left.R_{m}\right)$, explícitamente así lo señalan: tasa libre de riesgo, riesgo sistémico del sector y prima de riesgo de mercado, respectivamente.

La primera inquietud epistemológica que surge, entonces, al medir el riesgo de las acciones de una empresa es ¿por qué dicho elemento se mide de esa forma? En otras palabras, ¿por qué el riesgo se define matemáticamente y no de alguna otra manera?

La respuesta a esta interrogante la define la doctrina epistemológica del positivismo.

El positivismo descansa en la metodología racionalista, según la cual, a través de un proceso de argumentación puramente lógico, se concluye en una verdad necesariamente cierta. Los positivistas construyen su base doctrinal a partir de definiciones y axiomas propios dentro de un sistema cerrado de análisis. A este proceso le denominan teoría científica.

Según Comte (2018 [1876], p. 174), la evolución del conocimiento científico pasa por tres estadios distintos: uno teológico o ficticio, el cual considera provisional; uno metafísico o abstracto, de naturaleza irracional; y finalmente uno positivo o científico, que para él es el último y definitivo.
Así, pues, el positivismo, que no concibe otro conocimiento que no sea el conocimiento científico a través del método científico, constituye el paradigma epistemológico de nuestro tiempo y determina que la tasa de descuento relevante $\left(K_{e}\right)$ que define el riesgo de las acciones de la empresa sea calculada o medida a través de una ecuación matemática.

\section{Las finanzas neoclásicas}

El positivismo dio paso al desarrollo de las Neoclassical Finance, que representan la corriente principal en las finanzas actuales. Las finanzas neoclásicas (FN) se conciben a sí mismas como una ciencia positiva cuyos modelos deben ser juzgados según el criterio de falsación de Popper (1991), según el cual, contrastar una teoría significa intentar refutarla mediante un contraejemplo. Si no es posible refutarla, dicha teoría queda corroborada y puede ser aceptada provisionalmente, pero no verificada; es decir, ninguna teoría es absolutamente verdadera, sino, a lo sumo, no refutada.

Las FN descansan en los siguientes principios o pilares: "la eficiencia del mercado, las fuerzas del arbitraje, los procesos de selección de mercado y el retorno esperado como compensación por el riesgo sistemático" (Ross, 2002, p. 124). De acuerdo con este núcleo esencial de pensamiento, los mercados financieros se encuentran siempre en equilibrio, las fuerzas arbitrales hacen más eficientes los mercados y los precios son solo producto del riesgo sistemático (o sistémico) (Husserl, 2005, p. 115).

Por tanto, se concluye que las FN tienen una esencia cerrada, exclusiva, determinista e individualista que no permite, a diferencia de su corriente alterna (las finanzas conductuales) la participación o interacción con sistemas sociales, lo que, finalmente, alteraría los fenómenos tal como son analizados, según sus críticos, debido a su propio ostracismo teórico.

Durante la década de 1970, se impuso dentro de las FN la hipótesis de los mercados eficientes (HME) desarrollados por Markowitz y el modelo de equilibrio de activos financieros (CAPM) propuesto por Sharpe.

El aporte de la HME se cristaliza en la denominada línea del mercado de capitales, que articula el rendimiento esperado de un portafolio cualquiera con el nivel de su riesgo sistémico a través del coeficiente beta $(\beta)$. El modelo, conocido como el capital asset pricing model (CAPM), postula que los inversionistas solo pueden obtener rentabilidades superiores al mercado si asumen un nivel de riesgo sistémico mayor que dicho mercado, es decir, si invierten, según el aporte de Sharpe, endeudándose en la proporción del riesgo sistémico e invirtiendo sus propios recursos y los del préstamo sobre el índice de bolsa. 
Las FN postulan que los precios de los activos financieros tienen como ancla el valor presente neto de sus flujos futuros ajustados por riesgo. El aporte de la HME consiste, de este modo, en explicitar el concepto de ajustado por riesgo: tal ajuste se da a través del parámetro beta $(\beta)$. La evidencia empírica durante la década de 1970 mostró que el rendimiento obtenido por los portafolios estaba estrechamente relacionado con su beta.

El CAPM y la HME se mantienen vigentes, a pesar de que Fama y French (2004), después de un estudio comprensivo de más de cuarenta años, concluyeron que la relación existente entre la beta $(\beta)$ y el rendimiento $\left(R_{e}\right)$ es plana. De acuerdo con Kuhn (2006), la ciencia se construye sobre la base de paradigmas que hacen caso omiso de pequeñas anomalías en provecho de una construcción abstracta y general capaz de abarcar y de explicar un gran número de casos observados. Las FN no constituyen un contraejemplo y, más bien, han dado respuesta o resuelto todas las observaciones o críticas al CAPM dentro del mismo modelo, incluida la inexistencia de mercados completos.

Por otro lado, la HME se presume superior frente a otras escuelas alternativas de corte técnico, al haber hecho suyos los postulados tanto de Cassirer (1977) como de Koyré (1997):

La hipótesis verdadera no designa nada distinto a un principio y medio de medida. Ella no interviene una vez los fenómenos conocidos para adjuntarles a posteriori una presunción respecto de sus fundamentos absolutos, lo que ella hace es contribuir a hacer posible este orden mismo... la hipótesis verdadera indica el itinerario que nos permite acceder, a partir de la multiplicidad sensible de las impresiones, a la multiplicidad intelectual de la medida y de los números... el fenómeno no numerado y medido de ninguna manera aparece como el punto de partida incontestable inmediatamente dado y garantizado, sino que es el resultado de ciertas operaciones conceptuales de las cuales se trata de asegurar el ajuste de todos los detalles. (p. 105)

Para Koyré (1997), ni el empirismo ni la filosofía experimental llevan a algo positivo, puesto que considera que no se puede renunciar a la tarea del conocimiento por más inaccesible que parezca. Asimismo, este autor insiste en perseguir la verdad para el progreso de la ciencia y considera que "las grandes revoluciones del siglo XX han sido eminentemente teóricas, cuyo resultado no consistió en relacionar mejor entre ellos los datos de la experiencia, sino en adquirir una nueva concepción de la realidad profunda subyacente en esos datos" (Koyré, 1997, p. 75).
Como corolario del Modelo CAPM, dentro de las FN, la rentabilidad esperada a las acciones queda determinada por la siguiente expresión:

$$
\mathrm{Re}=\mathrm{Rf}+\beta(\mathrm{Rm}-\mathrm{Rf})
$$

El modelo CAPM se utiliza para calcular la tasa de descuento al valorar empresas, en general, y una Pyme, en particular. Además de utilizar el CAPM, la mayoría de expertos está de acuerdo en hacer ajustes a la tasa de descuento de una Pyme por su tamaño y en hacerlo por la iliquidez de la acción (falta de negociación), en contraste con Fernández (2017) que está completamente en desacuerdo en ambos casos, señalando que el tamaño no es siempre una fuente de riesgo en todos los sectores dado que hay empresas pequeñas con menos riesgo que otras más grandes. Por otro lado, no le parece que la iliquidez afecte al valor de la empresa cuando las acciones de una empresa no cotizada tienen comprador seguro, ya sea porque los estatutos de la empresa así lo establecen o porque un accionista quiera convertir su deuda en acciones.

Fernández (2017) descarta utilizar el CAPM por inconsistente y falto de predictibilidad, y propone una fórmula más sencilla para el cálculo de la rentabilidad exigida a las acciones:

$$
\mathrm{Ke}=\mathrm{Rf}+\mathrm{PRA}
$$

Donde:

\section{Ke: Rentabilidad exigida a las acciones}

Rf: Tasa Libre de Riesgo

PRA: Prima por Riesgo de la Acción

Señalando que como los flujos de caja para disponibles para el accionista tienen más riesgo que los bonos del Estado y la deuda emitida por la compañía o la deuda adquirida directamente del sistema financiero, la rentabilidad exigida a las acciones o costo de las acciones deberá ser mayor, tanto a la tasa libre de riesgo (Rf), como a la rentabilidad exigida a la deuda (Kd) (Fernández, 2017). Es cuestión de experiencia, conocimiento y sensatez, el establecer la prima por riesgo de la acción (PRA). No se necesita, ni se debe utilizar betas históricas, ni hacer ningún ajuste ni a la prima de riesgo de mercado ni a la tasa libre de riesgo.

Por el contrario, el modelo de valoración de empresas públicas y privadas de Damodaran (2006) utiliza el CAPM, proponiendo, además, ajustes matemáticos, tanto a la tasa libre de riesgo, como a la prima de riesgo de mercado, de acuerdo con el riesgo país, la primera, y de acuerdo con el riesgo explícito de la empresa, la segunda. En ambos casos, su metodología pasa por ajustes al modelo CAPM. 
Específicamente, Damodaran (2006) propone, para la tasa de descuento relevante o rentabilidad exigida a las acciones, lo siguiente:

$$
\mathrm{Ke}=\mathrm{Rf}+\mathrm{Rs}+\beta \mathrm{x}(\mathrm{Rm}-\mathrm{Rf}) \mathrm{x} \delta / \delta^{*}
$$

Donde:

$\mathrm{ke}=$ Rentabilidad exigida a las acciones

$\mathrm{Rf}=$ Tasa libre de riesgo local (Bono Soberano local)

Rs $=$ Spread de los bonos en dólares locales

Rf + Rs = Tasa Libre de Mercado Perú

$\beta=$ Beta estimado de la industria

$\mathrm{E}(\mathrm{Rm}-\mathrm{Rf})=$ Prima de riesgo de mercado de EE. UU.

$\delta=$ Desviación estándar de los rendimientos mensuales de la bolsa de valores local

$\delta^{*}=$ Desviación estándar de los rendimientos mensuales de los bonos del mercado local

En este modelo, la prima de riesgo del mercado se mide con la prima implícita de riesgo de mercado (PRIMI) obtenida del modelo de descuento de dividendos (Gordon-Shapiro).

De acuerdo con Damodaran (2006), la prima de riesgo de mercado debe ser ajustada por el nivel de riesgo específico de la empresa. Este riesgo específico dependerá también, al igual que la tasa libre de riesgo (Rf), del país donde se encuentre la empresa. Para Damodaran, no solo debe ajustarse la tasa libre de riesgo (Rf) por el diferencial (spread) que existe entre los rendimientos de los bonos locales y norteamericanos emitidos en la misma moneda. La prima de riesgo de mercado también debe ajustarse, según la volatilidad relativa del mercado accionario de Estados Unidos versus la volatilidad relativa del mercado de bonos del país donde se está midiendo la prima de riesgo de mercado.

\section{Las finanzas conductuales}

Las finanzas conductuales (FC) o finanzas del comportamiento, a diferencia de las finanzas neoclásicas, contemplan la incorporación de las ciencias sociales, concretamente de la psicología y la sociología, en el análisis de las decisiones financieras.

Las FC contradicen la mayor parte del fundamentalismo de las FN, especialmente la idea de la hipótesis de los mercados eficientes (HME). Así, este tipo de finanzas sugieren que hay suficiente evidencia para desestimar el paradigma vigente de que el mercado es eficiente y trabaja con personas racionales. En ese sentido, dicho paradigma no sería el adecuado para describir el comportamiento financiero de los diferentes actores (Elton, Gruber y Bused, 2004). De acuerdo con las FC, todas las personas llevan una carga emocional cuando toman decisiones y ello no es diferente con las decisiones financieras. En consecuencia, los modelos financieros son incapaces de reproducir y explicar esta realidad (Jensen y Meckling, 1994).

Las bases epistemológicas de las FC se remontan y apoyan en la teoría del conocimiento de Husserl (2005), quien plantea que el hombre es capaz de distinguir entre una experiencia auténtica y otra engañosa a través de su conciencia, de la misma manera que discernimos entre lo verdadero y lo falso. Así, en una primera instancia, Husserl se pregunta cómo toda esa actividad que se desarrolla en el umbral de la conciencia de cada quien puede volverse una experiencia objetiva, pero inmediatamente después se responde que esa pregunta es un contrasentido, pues, al ser hombres naturales, no tenemos derecho a plantearnos tal interrogante, ya que se trata de una cuestión trascendental. La simple percepción del mundo y de que pertenecemos a él debería hacernos entender que el valor de esa percepción ya se presupone en el planteamiento del problema, por lo que interviene en el sentido mismo de la pregunta.

Para Husserl (2013), la misión de la ciencia es alcanzar verdades universalmente válidas y considera que la eventual crisis de las FN se debe a que en ciencia se trata de medir cuán saturados de intuición están los contenidos de significación. Al contrario, según él, dicha intuición debería ser la vara con la que se mida la ciencia. De esta forma, se debería analizar si los conceptos esbozados, recogidos por cualquier fuente (experiencia u otra), pueden ser contrastados con la realidad. Por lo tanto, todos estos elementos, que no se cumplen en las teorías financieras vigentes, habrían dado lugar a la eventual crisis en las finanzas.

En última instancia, Husserl (2013) señala que el proceder correctamente, desde el punto de vista científico, supone hacer propuestas de ideas dentro de un modelo y proceso originalmente intuitivo que combine ideas simples que, a su vez, formen ideas complejas.

Así pues, el aporte de Husserl a la teoría del conocimiento es ser el primero en haber incorporado la intuición como fuente de conocimiento, concibiéndola como un conocimiento directo, inmediato y evidente, sin intervención de la deducción o del razonamiento.

\section{CONCLUSIONES}

Las principales inquietudes filosóficas y epistemológicas para abordar el objeto de estudio de este artículo se revelan en la metafísica y ética aristotélicas: las finanzas como disciplina pertenecen al campo de la técnica $y$, 
como tales, deben buscar la verdad como fin último. En segundo lugar, surge una reflexión respecto al riesgo: ¿por qué este se mide a través de una ecuación matemática y no de otra manera? Por lo hasta aquí expuesto, se concluye que esto se debe a la vigencia del positivismo como paradigma epistemológico de nuestros tiempos, el cual ha dado paso al desarrollo de las finanzas neoclásicas (FN). No obstante, a partir de la década de 1970, surgieron las finanzas conductuales (FC), que estaban provistas de una base epistemológica diferente: la fenomenología de Husserl. Empero, a pesar de la crítica de Husserl a las FN, el modelo de valoración de empresas propuesto en este artículo está enmarcado dentro de dichas finanzas. A continuación, se explica las razones de dicha decisión.

Primero, siguiendo a Kuhn (2006), para analizar un paradigma y su historia se debe manifestar la integridad histórica de esa misma teoría en su propia época. Así, se concluye que las FN son producto de su tiempo (positivismo), están vigentes y no existe, hasta este momento, un nuevo paradigma que las reemplace. Segundo, siguiendo a Popper (1991), creemos que la psicología no es aplicable a las finanzas debido a su falta de método científico y rigor. Finalmente, se descarta la aplicación de una teoría del conocimiento tan atractiva y compleja como la fenomenología de Husserl, debido a su falta de aplicabilidad a los modelos financieros.

En ese sentido, se propone utilizar, desde las FN, la razón, la experiencia y la sensatez en un modelo de valoración por descuento de flujo de fondos para Pymes. Con todo, luego de reconocer la eventual supeditación al racionalismo como base para el desarrollo de este modelo, se debe adoptar una posición final sobre la opinión de los expertos: por un lado, Damodaran (2006) sugiere ajustes matemáticos en la formulación de la tasa de descuento utilizada en un modelo de valoración con el CAPM; por el otro, Fernández (2017) propone no utilizar el CAPM por su falta de adecuación a la realidad.

Dentro de la aceptación de un racionalismo más amplio que el positivista, se conviene con Aristóteles (2014b), en un regreso a la razón práctica o juicio moral para tomar decisiones. De acuerdo con él, las finanzas como técnica, no tienen la perfección de las ciencias exactas. Más bien, requieren tanto de la razón práctica como de la razón técnica, bajo el supuesto de que los temas técnicos comprenden también cuestiones morales. En este sentido, debe entenderse la razón más como una apertura a la verdad que como una búsqueda de una certeza individual. No hay problemas realmente con las técnicas, sino con el pensamiento tecnicista vigente. No hay por qué matematizar más las fórmulas de cálculo ya existentes. Es cuestión, nuevamente, solo de conocimiento, experiencia y sensatez, el poder valorar adecuadamente una empresa en general y una Pyme, en particular. Por ello, se prefiere la posición de Fernández a la de Damodaran para optar por un modelo de valoración de empresas.

\section{REFERENCIAS}

Aristóteles. (2014a). Ética a Nicómaco. Madrid: Alianza Editorial.

Aristóteles. (2014b). Metafísica. Madrid: Alianza Editorial.

Cassirer, E. (1977). Sustancia y función: elementos para una teoría del concepto. Paris: Minuit.

Comte, A. (2018 [1876]). System of Positive Polity. Nueva York: Franklin Classics Press.

Damodaran, A. (2006). Damodaran on Valuation. Nueva Jersey: John Wiley \& Sons, Inc.

Elton, E., Gruber, M. y Bused, J. ( 2004). Are Investor Rational? Choices among Index Funds. The Journal of Finance, 59(1), 261-288.

Fama, E. y French, K. (2004). The Capital Asset Pricing Model: Theory and Evidence. Journal of Economic Perspectives, 18(3), 25-46.

Fernández, P. (2017). Valoración de empresas y sensatez. Madrid: Universidad de Navarra.

Husserl, E. (2005). Meditaciones cartesianas. México D.F.: Fondo de Cultura Económica.

Husserl, E. (2013). Filosofía primera.Tomo 1: Historia crítica de ideas. México D.F.: Fondo de Cultura Económica

Jensen, M. y Meckling, W. (1994). The Nature of Man. Nueva Jersey: Wiley \& Sons.

Koyré, A. (1997). Estudios de historia del pensamiento científico. Madrid: Siglo XXI Editores.

Kuhn, T. (2006). La estructura de las revoluciones científicas. Madrid: Fondo de Cultura Económica.

Popper, K. (1991). La lógica de la investigación científica. México D.F.: Red Editorial Iberoamericana.

Ross, S. (2002). Neoclassical Finance. Nueva Jersey: Princeton University Press. 